\title{
Surface Charge and Electrostatic Spin Crossover Effects in $\mathrm{CoN}_{4}$ Electrocatalysts
}

\author{
Zhiyao Duan and Graeme Henkelman*
}

Cite This: ACS Catal. 2020, 10, 12148-12155

Read Online

\section{ACCESS | Lلlll Metrics \& More | 回 Article Recommendations | st Supporting Information}

ABSTRACT: Carbon materials doped with nitrogen and $3 \mathrm{~d}$ transition metals have attracted a great deal of interest for catalyzing electrochemical reactions such as water splitting, oxygen reduction, and carbon dioxide reduction. Here, we employed density functional theory to study $\mathrm{Co}-\mathrm{N}$-doped carbon as electrocatalysts for the oxygen reduction and oxygen evolution reactions. Specifically, we investigated the interplay among adsorption energies, the spin state of the $\mathrm{CoN}_{4}$ active center, and the applied potential. We found that adsorption energies strongly depend on both the applied potential and the spin state of the Co center. Furthermore, spin state transitions induced by the applied potential also play an important role in determining the adsorption energies. This effect originates from a different potential of zero charge and capacitance of each spin state.

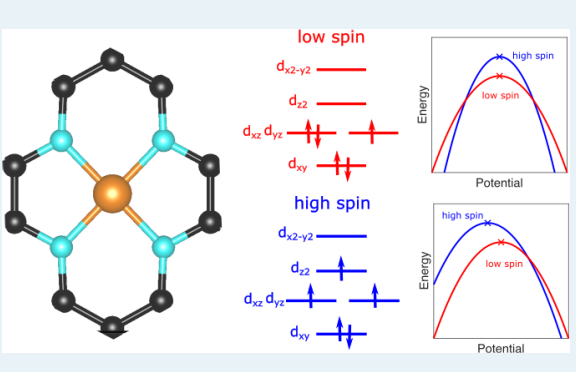

KEYWORDS: Co- and $\mathrm{N}$-doped carbon, spin crossover effect, oxygen reduction reaction, oxygen evolution reaction, $p H$-dependent activity, density functional theory, constant-potential condition

\section{INTRODUCTION}

The electrocatalytic oxygen reduction reaction (ORR) and oxygen evolution reaction (OER) are two key processes for proton-exchange membrane fuel cells, ${ }^{1}$ artificial photosynthesis, ${ }^{2}$ and metal-air batteries. ${ }^{3}$ Electrocatalysts with high activity and durability toward these reactions are critically important for bringing these technologies toward wide deployment in our society. The state of the art catalysts for these reactions, however, contain precious metals such as $\mathrm{Pt}$ for the ORR and $\mathrm{Ir} / \mathrm{Ru}$ oxides for the OER; their high costs and scarcity reduce the economic feasibility of these technologies in comparison to traditional energy industries based on fossil fuels. Replacing precious-metal catalysts with catalysts made of cheaper and more earth abundant elements is an active pursuit by researchers worldwide. ${ }^{4-6}$ Among various noble-metal-free formulations, $3 \mathrm{~d}$ transition-metal and nitrogen-codoped carbon materials (M-N-C) are among the most promising groups of catalysts for both the ORR ${ }^{4}$ and OER. ${ }^{6}$ Specifically, Fe-, ${ }^{7,8} \mathrm{Co}-$, $^{9,10}$ and $\mathrm{Mn}-\mathrm{N}-\mathrm{C}^{11-13}$ have been demonstrated to exhibit high ORR and OER activities. Perhaps the most likely active sites of these M-N-C catalysts are single-atom $\mathrm{MN}_{4}$ centers embedded in the carbon matrices. ${ }^{11,14,15}$

In studies of $\mathrm{M}-\mathrm{N}-\mathrm{C}$ catalysts, density functional theory (DFT) calculations have played an important role in facilitating the structural characterization of known active sites and also evaluating the activity of proposed active sites. $^{16-18}$ The latter is achieved by coupling DFT-calculated binding energies of ORR and OER intermediates, i.e. OOH*, $\mathrm{OH}^{*}$, and $\mathrm{O}^{*}$, with the famous computational hydrogen electrode method, ${ }^{19}$ which then enables the prediction of overpotentials of the ORR and OER at the active sites of interest. Although it has been very successful in guiding the development of Pt-based alloy catalysts for the ORR, ${ }^{20,21}$ this method oversimplifies the physics of the electrochemical interface under the reaction conditions, leading to deviations from experimental results for M-N-C catalysts. For example, the onset potentials for the ORR over $\mathrm{FeN}_{4}$ and $\mathrm{MnN}_{4}$ are both predicted to be $\sim 0.58 \mathrm{~V}$ vs the reversible hydrogen electrode (RHE), whereas the experimental values are $0.2-0.3$ $\mathrm{V}$ higher than the predictions. ${ }^{22}$ A similar discrepancy between theory and experiment exists for $\mathrm{CoN}_{4} \cdot{ }^{9,18}$ In order to deepen our understanding of the electrocatalytic properties of M-N-C catalysts, it is important for theorists to track down the missing physics in the DFT calculations that lead to the discrepancies.

For one thing, in the aforementioned DFT studies, the binding energies of reaction intermediates are calculated assuming constant-charge conditions, in which the total number of electrons before and after the adsorption remain constant. This assumption is not suitable for electrocatalysis because the electrode potential is typically held constant, leading to charge differences at the electrochemical interface before and after adsorption. It has recently been pointed out

Received: June 3, 2020

Revised: September 21, 2020

Published: September 25, 2020 
that the charge differences caused by the constant-potential condition can have a more significant effect on carbon materials than on metal electrodes due to a limited density of states of the former. ${ }^{23}$ When constant-potential conditions are considered in DFT calculations, the calculated binding energies can have a strong dependence on the applied potential, resulting in a more accurate understanding of the electrocatalytic behaviors. For example, the potential-dependent binding energies under constant-potential conditions have been adopted to explain $\mathrm{pH}$-dependent electrocatalytic activities of $\mathrm{Au}$ for $\mathrm{CO}$ electro-oxidation and ORR. ${ }^{24,25}$

Second, it is surprising that the spin states of the M-N-C catalysts are often not investigated in detail when binding energies are calculated. ${ }^{16}$ Ideally, all possible spin states, i.e. low-spin (LS), intermediate-spin (IS), and high-spin (HS) states, should be examined to determine the most stable state in order to obtain accurate binding energies. Moreover, it is well-known that the spin state of transition-metal complexes can change in response to external stimuli, including temperature, pressure, and photoexcitation, via spin crossover. ${ }^{26}$ At the electrochemical interface, the $\mathrm{MN}_{4}$ active sites are subject to strong electric fields in the electric double layer. The spin state of the $\mathrm{MN}_{4}$ centers might also undergo spin crossover in response to the electric field, as modulated by the applied potential. The control of spin transitions by an applied electric field has already been demonstrated and has important implications for molecular spintronics. ${ }^{27,28}$ In electrocatalysis, however, the electrostatic spin crossover effect has seldom been studied for M-N-C catalysts.

Here, we employed DFT to calculate the binding energies of reaction intermediates of the ORR and OER on the $\mathrm{CoN}_{4}$ active center under constant-potential conditions. Specifically, we systematically investigated the magnetic states of bare and adsorbed $\mathrm{CoN}_{4}$ centers as a function of electrode potential. We found that the applied potential can induce spin crossover in the $\mathrm{CoN}_{4}$ center because each spin state responds differently to the electric field in the electric double layer. As we show, the electrostatic spin crossover effect plays an important role in the electrocatalytic activities of $\mathrm{CoN}_{4}$ toward the ORR and OER.

\section{COMPUTATIONAL METHODS}

Spin-polarized DFT calculations with a plane-wave basis set were performed using the Vienna $a b$ initio simulation package (VASP). ${ }^{29-31}$ Electronic exchange and correlation were described using the Perdew-Burke-Ernzerhof functional. ${ }^{32}$ Core electrons were treated within the projector augmented wave framework. ${ }^{33}$ In all calculations, the energy cutoff of the plane wave basis set was $400 \mathrm{eV}$. The $\mathrm{DFT}+U$ method was applied to the $3 \mathrm{~d}$ orbitals of Co to correct for artificial electron self-interaction. $^{34}$ A value of $U_{\text {eff }}=3.3 \mathrm{eV}$ was used to reproduce the experimental heat of formation of Co oxides. ${ }^{35}$

Slab models consisting of five graphene layers in a $p(4 \times 4)$ supercell were employed to model clean and adsorbatecovered $\mathrm{CoN}_{4}$ embedded in graphite surfaces. The middle layer was fixed during structural relaxation. Symmetrical slabs with adsorbates on both surfaces of the slab models were used to eliminate any electric dipoles. The total surface area was 167.84 $\AA^{2}$ for the model. A $20 \AA$ vacuum gap was used to eliminate interactions between periodic images perpendicular to the slab surface. The Brillouin zone integration in $k$ space was performed on a $10 \times 10 \times 1 k$-point mesh sampled using the Monkhorst-Pack scheme. ${ }^{36}$ Optimized structures were obtained by minimizing the forces on each ion until they fell below $0.05 \mathrm{eV} / \AA$. The occupation matrix control plugin for VASP, developed by the Watson group, was employed to generate initial structures according to different metastable magnetic states. ${ }^{37}$ This was achieved by constraining the $\mathrm{CoN}_{4}$ spin state by setting the occupation matrix of d-electron localization and then relaxing the geometry. The constraint on the spin state was subsequently removed, and the system was allowed to relax toward the targeted metastable state.

The applied voltage to the electrochemical interface was simulated by adding or removing electrons to the supercell. The charged slab together with the compensating homogeneous background charge (automatically added by VASP) polarized the electrode/electrolyte interface. The aqueous environment of the electrolyte was treated with a continuum dielectric model as implemented by the Hennig group in the VASPsol code. ${ }^{38,39}$ The relative permittivity was set to 80 to model an aqueous electrolyte. The cavity setting in VASPsol was turned off by setting TAU $=0$. The potential-dependent free electrochemical energy of the electrode/electrolyte interface can be calculated as ${ }^{40,41}$

$$
E_{\text {free }}(q)=E_{\mathrm{SCF}}(q)+E_{\text {corr }}(q)-q \phi_{q}(f)
$$

where $q$ is the excess number of electrons, $E_{\mathrm{SCF}}$ is the selfconsistent energy directly obtained from DFT calculations, $E_{\text {corr }}$ is the correction to the $E_{\mathrm{SCF}}$ due to the use of homogeneous background charge, and $-\phi_{q}(f)$ which is the work function of the charged slab. $E_{\text {corr }}$ is calculated using the average electrostatic potential of the supercell $\left\langle\bar{V}_{\text {tot }}\right\rangle$ :

$$
E_{\text {corr }}=\int_{0}^{q}\left\langle\bar{V}_{\text {tot }}\right\rangle \mathrm{d} Q
$$

The electric potential of the charged slab with $q$ excess electrons referenced to the SHE is calculated as

$$
U_{q}(V / \text { SHE })=-4.6-\phi_{q}(f) / \mathrm{eV}
$$

where $4.6 \mathrm{~V}$ is the work function of the $\mathrm{H}_{2} / \mathrm{H}^{+}$couple under standard conditions. In order to model the charged interfaces at different applied potentials, for each modeled structure, calculations were performed at charges of $-2.0 \mathrm{e}$ to $+2.0 \mathrm{e}$ with increments of $+0.5 \mathrm{e}$. The free energy at the 11 charge values were then fit to a quadratic function to provide the free energy as a continuous function of potential. The quadratic form is consistent with a capacitor created by the charged-slab/ background-charge system, which takes the form

$$
E_{\text {free }}(U)=-\frac{1}{2} C\left(U-U_{0}\right)^{2}+E_{0}
$$

where $U_{0}$ refers to the potential of zero charge (PZC), $E_{0}$ is the energy at the PZC, and $C$ is the capacitance of the surface. From the fitted quadratic functions for the bare slab and slabs with adsorbates, the binding energies and reaction energetics as a function of electric potential were calculated.

The $\mathrm{pH}$ can also affect the adsorption energies of reaction intermediates by effectively changing the electric potential. Indeed, by changing the $\mathrm{pH}$ value, the electric potential on the standard hydrogen electrode (SHE) scale also change at a fixed potential on the reversible hydrogen electrode (RHE) scale according to the relation

$$
U_{\mathrm{RHE}}=U_{\mathrm{SHE}}+k_{\mathrm{B}} T \ln (10) \mathrm{pH} / e
$$



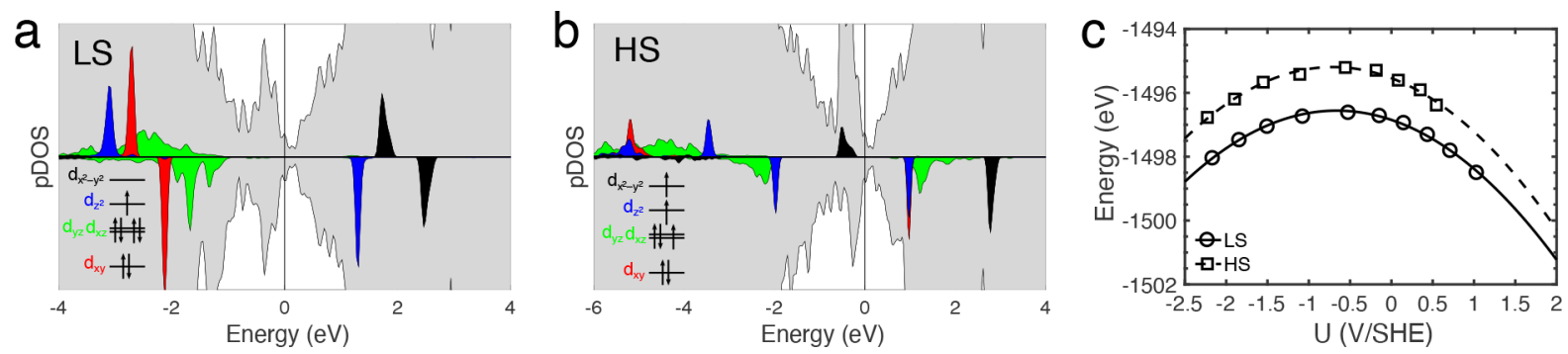

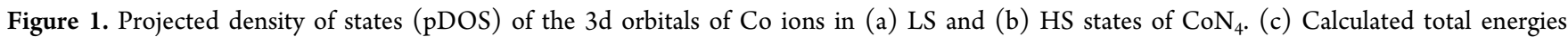
(points) and polynomial fits (lines) of LS and HS states of $\mathrm{CoN}_{4}$ as a function of potential. The shaded areas in (a) and (b) are the total DOS.
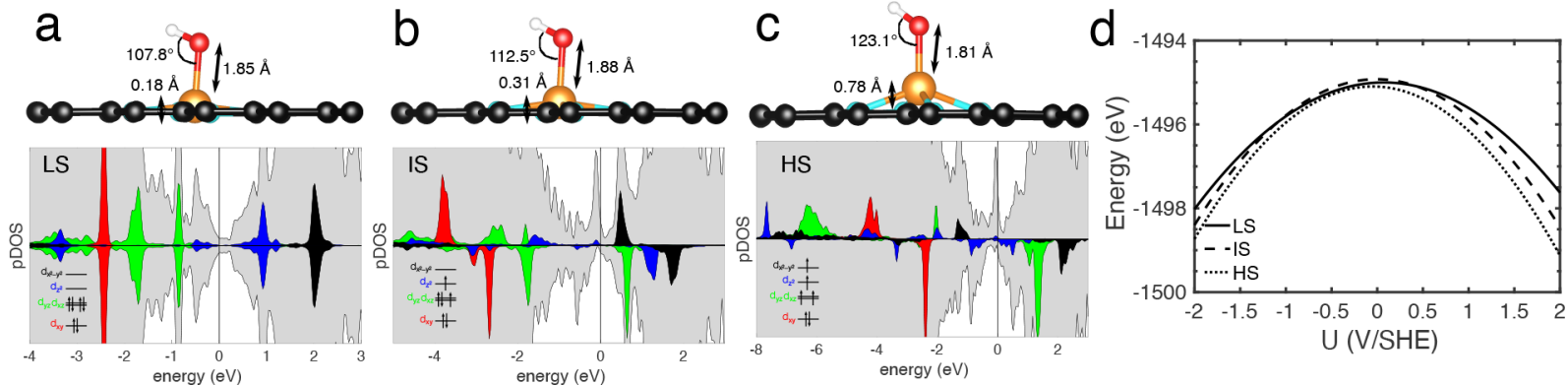

Figure 2. Atomic structures and pDOS of $3 \mathrm{~d}$ orbitals of Co ions in the (a) LS, (b) IS, and (c) HS states of *OH/CoN ${ }_{4}$ (d) Polynomial fits of various spin states of $* \mathrm{OH} / \mathrm{CoN}_{4}$ as a function of potential. The calculated total energy values can be found in the Supporting Information. The shaded areas in $(\mathrm{a})-(\mathrm{c})$ are the total DOS.

In this study, we evaluate $\mathrm{pH}$-dependent ORR activities on the RHE scale by applying different binding energies at the SHE scale at different $\mathrm{pH}$ values.

A computational hydrogen electrode method was employed to construct the free energy diagram of the electrochemical reactions. ${ }^{19,42}$ In this method, the binding energies of three intermediates $\left(* \mathrm{O}_{2} \mathrm{H}, * \mathrm{O}\right.$, and $\left.* \mathrm{OH}\right)$ as a function of applied potential were calculated using the potential-dependent total energies of the clean and adsorbed models. Specifically, the binding energies ( $\Delta E$ values) were calculated as follows:

$$
\begin{aligned}
& \Delta E_{* \mathrm{O}_{2} \mathrm{H}}(U)=\frac{1}{2}\left(E_{* \mathrm{O}_{2} \mathrm{H}}(U)-E_{*}(U)-4 E_{\mathrm{H}_{2} \mathrm{O}}+3 E_{\mathrm{H}_{2}}\right) \\
& \Delta E_{* \mathrm{O}}(U)=\frac{1}{2}\left(E_{* \mathrm{O}}(U)-E_{*}(U)-2 E_{\mathrm{H}_{2} \mathrm{O}}+2 E_{\mathrm{H}_{2}}\right) \\
& \Delta E_{* \mathrm{OH}}(U)=\frac{1}{2}\left(E_{* \mathrm{OH}}(U)-E_{*}(U)-2 E_{\mathrm{H}_{2} \mathrm{O}}+E_{\mathrm{H}_{2}}\right)
\end{aligned}
$$

The binding energies are halved because of the symmetrical slab model employed. The free energy corrections for $* \mathrm{O}_{2} \mathrm{H}$, $* \mathrm{O}$, and $* \mathrm{OH}$ are $0.4,0.05$, and $0.35 \mathrm{eV}$, respectively. ${ }^{19}$

\section{RESULTS AND DISCUSSION}

Bare $\mathrm{CoN}_{4}$. We examined the LS and HS states of $\mathrm{CoN}_{4}$. The projected density of states (pDOS) of the $3 \mathrm{~d}$ orbitals of Co ions of these two states and the corresponding schematic electronic structures are shown in Figure $1 \mathrm{a}, \mathrm{b}$, respectively. The $\mathrm{CoN}_{4}$ center has a square-planar geometry, which causes splitting of the degenerate $3 \mathrm{~d}$ states of Co into $\mathrm{d}_{x}{ }^{2}-y^{2}, \mathrm{~d}_{z}{ }^{2}$, doubly degenerate $\mathrm{d}_{x z}$ and $\mathrm{d}_{y z}$, and $\mathrm{d}_{x y}$ energy levels. The Co ion in $\mathrm{CoN}_{4}$ has a $3 \mathrm{~d}^{7}$ electron configuration. The LS state of $\mathrm{CoN}_{4}$ features the full occupancy of lower $\mathrm{d}_{x y}, \mathrm{~d}_{x z}$, and $\mathrm{d}_{y z}$ levels, leaving a single electron in the $\mathrm{d}_{z}{ }^{2}$ level. Hence, the LS state has a total spin of $S=1 / 2$. In the HS state, one electron in the $d_{x z}$ or $d_{y z}$ level gets excited to the $d_{x^{2}-y^{2}}$ level, which possesses a total spin of $S=3 / 2$. The d-orbital occupancy of $\mathrm{CoN}_{4}$ does not change as a function of potential; details can be found in Figure S5.

The total energies of the LS and HS states as a function of electrode potential are calculated and presented in Figure 1c. The potential-dependent energy of the HS and LS states are well represented by the parallel capacitor model and thus follow a parabolic function. The fitted parameters of the parabolic functions are given in Table S1. The PZCs and capacitances of the two states are similar. The energy gap between the LS and HS states is as high as $1.4 \mathrm{eV}$. Thus, no spin crossover is possible and the LS state is the ground state of $\mathrm{CoN}_{4}$ in the potential range of interest.

$* \mathrm{OH} / \mathrm{CoN} \mathrm{N}_{4}$. After $\mathrm{OH}$ adsorption on the $\mathrm{CoN}_{4}$ center, the Co ion transfers one more electron to $* \mathrm{OH}$. Consequently, the Co ion has the $3 \mathrm{~d}^{6}$ electron configuration. The electronic structures of the Co d orbitals in the LS, IS, and HS states of $* \mathrm{OH} / \mathrm{CoN}_{4}$ and their corresponding atomic structures without doped charge are shown in Figure $2 \mathrm{a}-\mathrm{c}$. The LS state of $* \mathrm{OH} /$ $\mathrm{CoN}_{4}$ has a total spin of $S=0$ since the unpaired electron on the $\mathrm{d}_{z}{ }^{2}$ orbital in the $\mathrm{CoN}_{4}$ LS state transfers to ${ }^{*} \mathrm{OH}$. The IS state features two unpaired electrons in the $\mathrm{d}_{x z}$ or $\mathrm{d}_{y z}$ and $\mathrm{d}_{z}{ }^{2}$ orbitals and thus has a total spin of $S=1$. In the HS state, four unpaired electrons occupy the $\mathrm{d}_{x z}, \mathrm{~d}_{y z} \mathrm{~d}_{z^{2}}$, and $\mathrm{d}_{x^{2}-y^{2}}$ orbitals, with a total spin of $S=2$. The potential-dependent d-orbital occupancies of LS, IS, and HS states can be found in Figure S5.

The atomic structures of $* \mathrm{OH} / \mathrm{CoN}_{4}$ vary with spin state, as shown in Figure 2. A most striking structural change between spin states is the location of the Co ion relative to the graphene plane. The Co ion sticks out of the plane as the spin state goes from LS to IS and HS. In the HS state, the Co ion lies above the plane by $0.78 \AA$. In addition, the adsorbed $* \mathrm{OH}$ sticks up 
more in the IS and HS states, which can be characterized by the $\angle \mathrm{CoOH}$ angle. The potential-dependent geometries of LS, IS, and HS states can be found in Figure S6.

The potential-dependent energies of the three spin states of $* \mathrm{OH} / \mathrm{CoN}_{4}$ are shown in Figure 2d. Only the fitted polynomial curves are shown in Figure $2 \mathrm{~d}$ for clarity; the calculated total energy points can be found in Figure S2, and the fitted parameters are summarized in Table S1. The energy differences among the three spin states are small at their respective PZCs. The HS is slightly more stable than the other states with a $0.2 \mathrm{eV}$ energy difference. The three spin states, however, respond to the applied electrode potential differently. The HS state becomes more stable than the other two states under potentials deviating from their respective PZCs. This is because the HS state has the largest capacitance in comparison to the other states, making its energy decrease more rapidly as a function of the applied potential.

The higher capacitance of the HS state of the $* \mathrm{OH} / \mathrm{CoN}_{4}$ partially originates from its higher quantum capacitance, as discussed below. The quantum capacitance was first introduced in 1988 in a pioneering work for designing novel transistors. ${ }^{43}$ The relevance between quantum capacitance and electrocatalysis of carbon materials has, however, seldomly been studied. ${ }^{44}$ It is well-known that, in a metal/electrolyte electrochemical interface, there is an electric potential drop $\phi_{\mathrm{dl}}$ across the interface caused by the surface charge and neutralizing ions in the electrolyte. Therefore, the electric double layer acts as a capacitor of capacitance $C_{\mathrm{dl}}$. When the metal electrode is replaced with carbon materials, due to their low DOS, the applied voltage can also cause band filling or depletion and a corresponding Fermi level shift. This effect results in a second capacitance in series to the electric capacitance in the double layer, which is called the quantum capacitance $\left(C_{\mathrm{q}}\right)$. In Figure 3, we plot potential profile

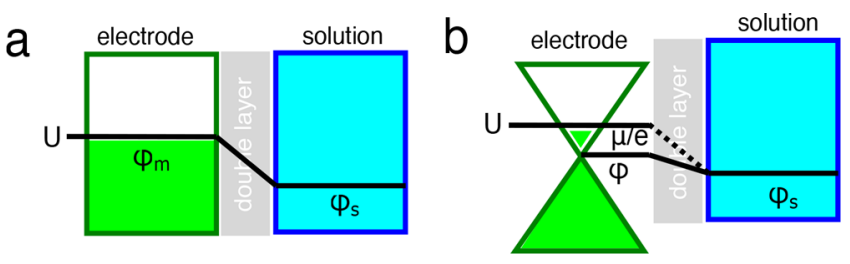

Figure 3. Potential profile diagram across the (a) metal/solution interface and (b) carbon/solution interface.

diagrams across the electrode/electrolyte interfaces comparing the traditional metal/electrolyte interface and carbon/electrolyte interface under an applied potential $U$. As seen from Figure 3, the electric potential of the metal electrode $\phi_{\mathrm{m}}$ equals the applied potential. In carbon (or in general for materials with low DOS) the applied potential is determined by the electric potential $\phi$ and the shift in electron chemical potential $\mu$, as seen in Figure $3 \mathrm{~b}$, and thus $U=\phi+\mu / e$. By differentiating the equation with respect to the charge density $q$, we can get

$$
1 / C=1 / C_{\mathrm{dl}}+1 / C_{\mathrm{q}}
$$

where $C$ is the total capacitance or electrochemical capacitance, $C_{\mathrm{dl}}$ is the traditional electric capacitance of the double layer, and $C_{\mathrm{q}} \equiv \mathrm{e}(d q / d \mu)$ is the quantum capacitance.

To calculate the chemical potential shift $\mu$, we integrate the total DOS to obtain the energy level that can accommodate the number of electrons as if the system has zero charge; the difference between this energy level and the Fermi energy is determined to be $\mu$. Then the electric potential drop $\phi$ can be determined by using $\phi=U-\mu / e$. The validity of this approach was tested against a metal electrode of $* \mathrm{OH} /$ $\operatorname{Pt}(111)$. We plotted the doped charge against $U, \phi$, and $\mu / e$ as shown in Figure S3. Obviously, the quantum capacitance q/ $(\mu / e)$ is infinitely large due to the large DOS of the metallic system and so the total capacitance coincides with the doublelayer capacitance. Similar plots for different spin states of $* \mathrm{OH} / \mathrm{CoN}_{4}$ are shown in Figure 4. The charge vs potential curves are fit with linear functions to obtain the slopes corresponding to the approximate capacitance values. It can be seen that $C_{\mathrm{q}}$ values for all the spin states have magnitudes comparable to the $C_{\mathrm{dl}}$ values due to the low DOS near the Fermi energy. Thus, the influence of the quantum capacitance cannot be ignored. Among the three spin states, the HS state has the largest $C_{\mathrm{q}}$ of $5.9 \mathrm{e} / \mathrm{V}$, which is consistent with its highest DOS at the Fermi energy, as shown in Figure 2. Consequently, the HS state should have the lowest potential drop due to quantum capacitance, which will lead to higher total capacitance, assuming that all spin states have the same $C_{\mathrm{dl}}$.

In addition to the higher $C_{\mathrm{q}}$ value for the HS state, the $C_{\mathrm{dl}}$ value of the HS state is also higher than those of the other two spin states. To determine the physical origin of the $C_{\mathrm{dl}}$ of the HS state, we studied the dipole moments associated with the $* \mathrm{OH} / \mathrm{CoN}_{4}$ center and their change caused by the applied potential. We are only concerned with the dipole moments along the $z$ direction, perpendicular to the interface. The dipole moment is calculated as $p_{z}=\sum_{i} q_{i}\left(z_{i}-z_{0}\right) . q_{i}$ and $z_{i}$ are charges and $z$ positions for $4 \mathrm{~N}, \mathrm{Co}, \mathrm{O}$, and $\mathrm{H}$ atoms at the $* \mathrm{OH} / \mathrm{CoN}_{4}$ center, respectively. The atoms are considered point charges and are assigned $-0.5 \mathrm{e},+3 \mathrm{e},-2 \mathrm{e}$, and $+1 \mathrm{e}$ charges for $\mathrm{N}, \mathrm{Co}, \mathrm{O}$, and $\mathrm{H}$ atoms, respectively. The change in dipole moments as a function of electric field (assuming a
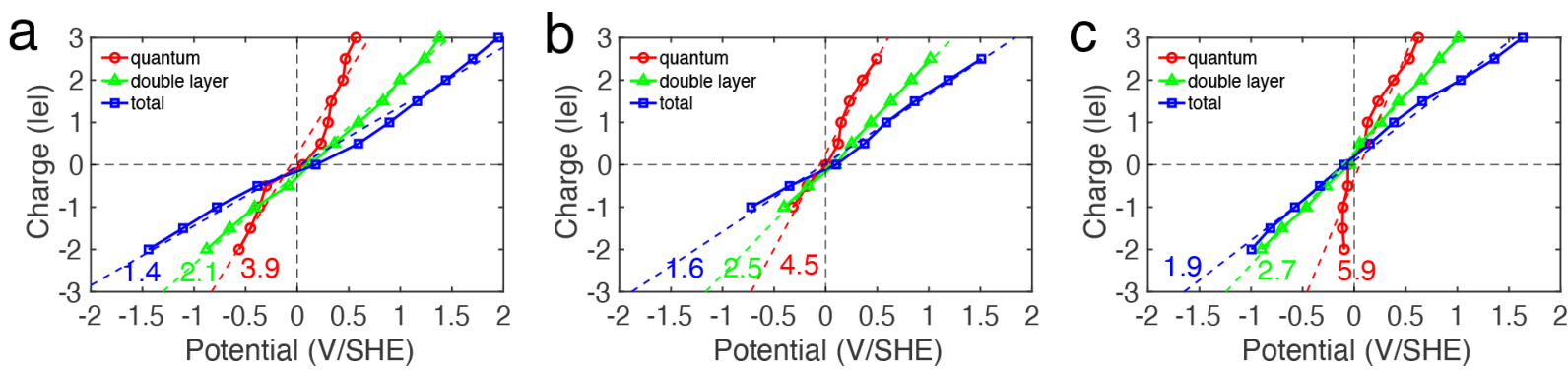

Figure 4. Doped charge as a function of $\mu / e, \phi$ (green), and $U$ (blue) for (a) LS, (b) IS, and (c) HS states of * OH/CoN 4 The calculated data are fit to linear functions to obtain the slopes as indicated. 
double layer width of $3 \AA$ ) for different spin states are shown in Figure 5. The calculated points are fit to linear equations, and

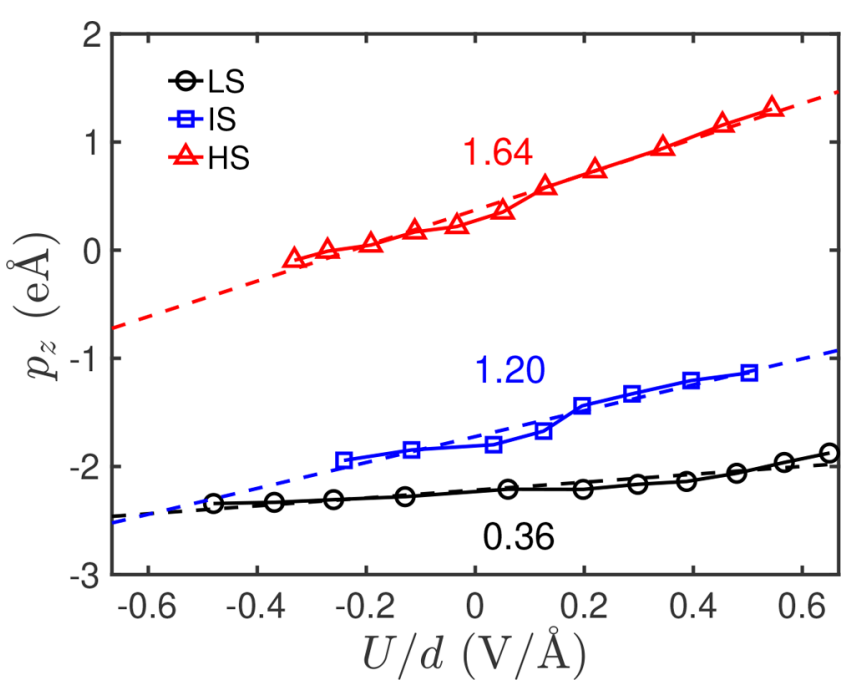

Figure 5. Electric dipole moments at the $* \mathrm{OH} / \mathrm{CoN}_{4}$ center as a function of interfacial electric field. The linear fit slopes are indicated.

the slope indicates the polarizability of the $* \mathrm{OH} / \mathrm{CoN}_{4}$ center. The polarizability of the different spin states follow the order HS > IS > LS, which is consistent with the order of $C_{\mathrm{dl}}$ capacitance of $* \mathrm{OH} / \mathrm{CoN}_{4}$ centers of different spin states. The high polarizability of the HS state originates primarily from the easy conformational rotation of $* \mathrm{OH}$ under an electric field.

$* \mathrm{O}_{2} \mathrm{H} / \mathrm{CoN}_{4}$. The atomic and electronic structures of the Co $3 \mathrm{~d}$ orbitals of $* \mathrm{O}_{2} \mathrm{H} / \mathrm{CoN}_{4}$ in the different spin states are shown in Figure $6 \mathrm{a}-\mathrm{c}$. The electronic structures of $* \mathrm{O}_{2} \mathrm{H} /$ $\mathrm{CoN}_{4}$ are similar to those of $* \mathrm{OH} / \mathrm{CoN}_{4}$. Structurally, the Co ion also rises up from the graphene plane in the HS state as in the case of $* \mathrm{OH} / \mathrm{CoN}_{4}$. Another major structural difference between different spin states is the orientation of the $* \mathrm{O}_{2} \mathrm{H}$ molecule, which can be characterized by the dihedral angle between the $\mathrm{OOH}$ and $\mathrm{CoOO}$ planes, as indicated by the red arc in Figure $6 a-c$. In the IS state, the dihedral angle is the largest, indicating that the $\mathrm{H}$ atom is pointing upward rather than lying flat, as in the LS state. The potential-dependent $\mathrm{d}$ orbital occupancies and geometries of LS, IS, and HS states can be found in Figures S5 and S7, respectively.

The potential-dependent energies of the three spin states have been calculated and are presented in Figure $6 \mathrm{~d}$. It can be seen that the PZCs of the LS and HS are nearly identical at about $0.06 \mathrm{~V}$. The total energy of the LS state is lower than the
HS state at the PZC. The HS state, initially less stable, becomes more stable than the LS state at high potential due to the higher capacitance of the HS state. The potentials for spin crossover are located at -1.2 and $1.5 \mathrm{~V}$. The higher capacitance of the HS state is due to its higher quantum capacitance in comparison to that of the LS state, as can be seen in Figure S4. The IS state, however, has a much lower PZC, $-0.45 \mathrm{~V}$ lower than those of the other two spin states. As a result of this PZC shift, the IS state becomes energetically favorable at potentials larger than $0.2 \mathrm{~V}$, as seen in Figure $6 \mathrm{~d}$.

So far, we have demonstrated that the spin crossover effect can be induced by the applied potential. The spin crossover can be realized in two different ways. First, when two spin states have the same PZCs but different capacitances, the spin state of higher capacitance can be more stabilized by the electric field due to the second-order Stark effect, as depicted in Figure 7a. Second, when the PZCs are different for the two

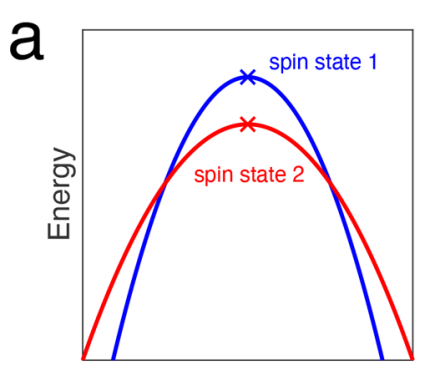

Potential

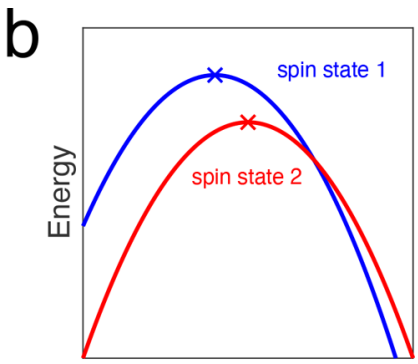

Potential
Figure 7. Schematics of two cases involving spin-state transitions.

spin states, one of the states can be stabilized more than the other state by the bias potential due to the first-order Stark effect, as shown in Figure $7 \mathrm{~b}$.

$* \mathrm{O} / \mathrm{CoN}_{4}$. The atomic structure of $* \mathrm{O} / \mathrm{CoN}_{4}$ is shown in Figure $8 \mathrm{a}$. The Co ion has a $3 \mathrm{~d}^{5}$ electronic configuration because two electrons transfer to $* \mathrm{O}$. The electronic structures of the LS and IS states of $* \mathrm{O} / \mathrm{CoN}_{4}$ are shown in Figure $8 \mathrm{~b}, \mathrm{c}$. The HS state is found to be unstable, since it relaxes to the IS state upon removing the occupancy matrix constraint. The potential-dependent energies of the LS and IS states are shown in Figure 8c. The IS state is the energetically favorable state in the potential range of interest. The potential-dependent $d$ orbital occupancies and geometries of LS and IS states can be found in Figures S5 and S8, respectively.

Binding Energy and ORR/OER Activity. The binding energies of ORR and OER reaction intermediates are calculated as shown in Figure 9. On the basis of the binding
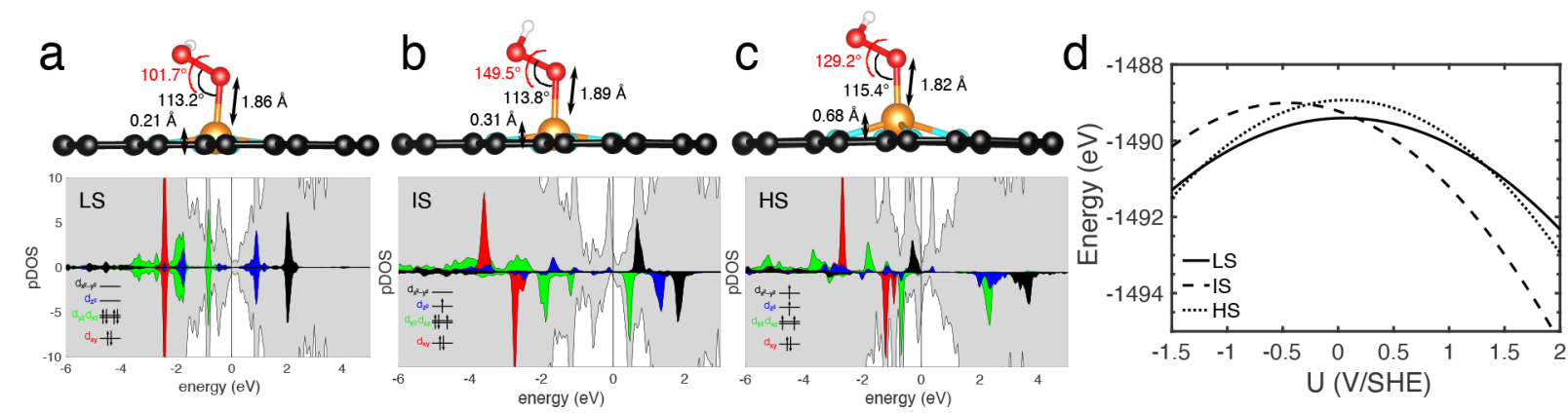

Figure 6. Atomic structures and pDOS of $3 \mathrm{~d}$ orbitals of Co ions in the (a) LS, (b) IS, and (c) HS states of $* \mathrm{O}_{2} \mathrm{H} / \mathrm{CoN}_{4}$. (d) Polynomial fits of various spin states of $* \mathrm{O}_{2} \mathrm{H} / \mathrm{CoN}_{4}$ as a function of potential. The calculated total energy values can be found in the Supporting Information. 

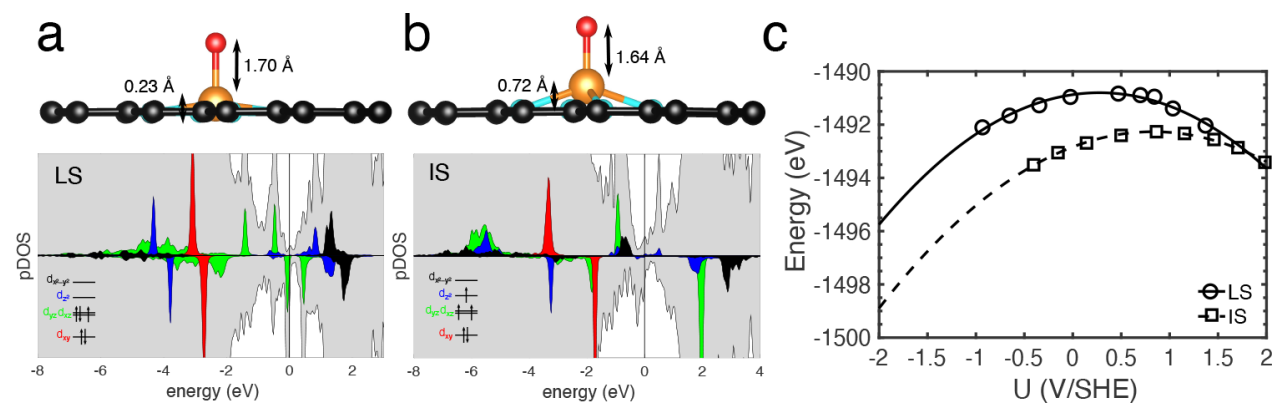

Figure 8. Atomic structures and pDOS of $3 \mathrm{~d}$ orbitals of Co ions in (a) LS and (b) IS states of $* \mathrm{O} / \mathrm{CoN}_{4}$. (c) Calculated total energies (points) and polynomial fits (lines) of the LS and IS states of $* \mathrm{O} / \mathrm{CoN}_{4}$ as a function of potential.

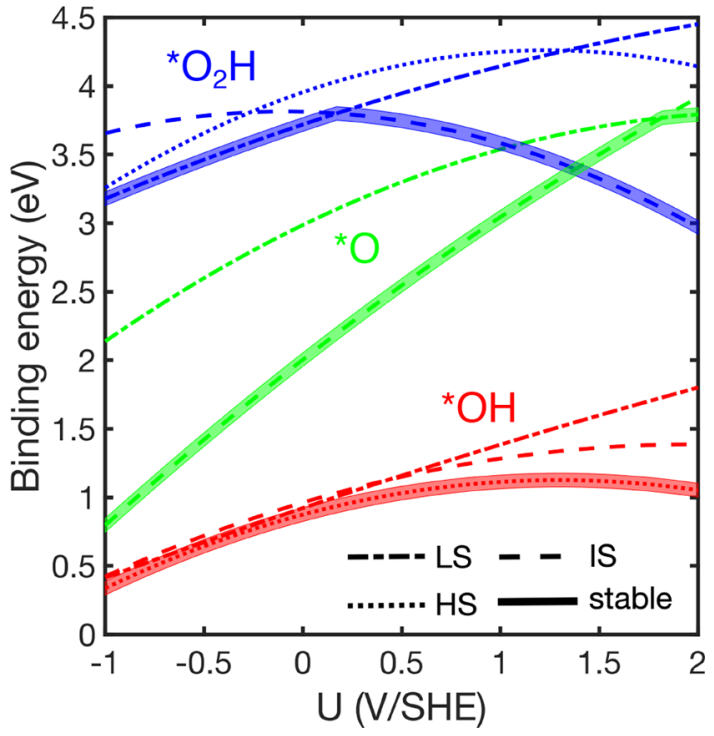

Figure 9. Binding energies of reaction intermediates as a function of electrode potential.

energies, the ORR and OER activities of $\mathrm{CoN}_{4}$ at $\mathrm{pH} 1$ and 13 are predicted using the hydrogen electrode method. The free energy diagrams constructed using the calculated binding energies are shown in Figure 10. The predicted onset potentials for the ORR in acid and alkaline electrolytes are 0.8 and $0.76 \mathrm{~V} / \mathrm{RHE}$, respectively. It should be noted that the
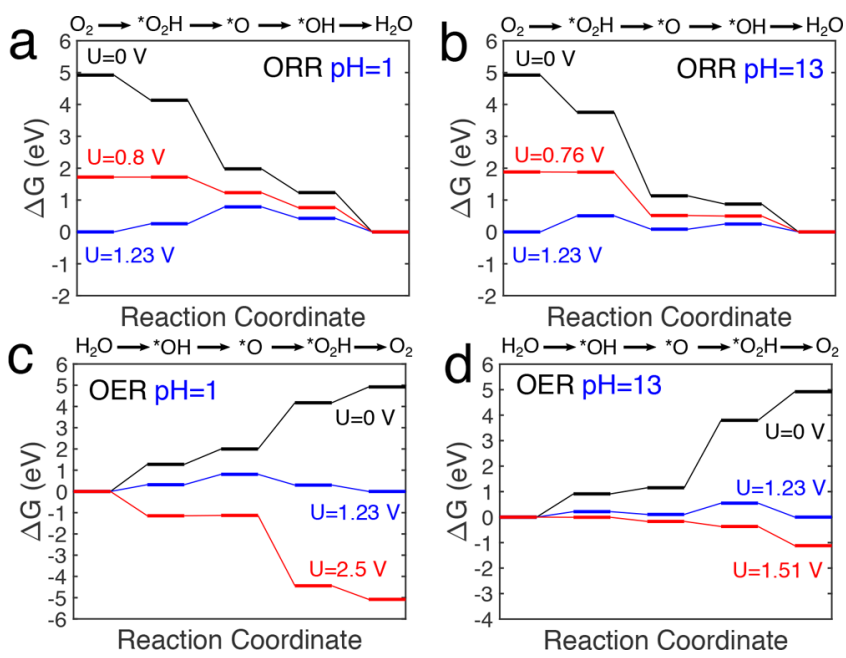

Figure 10. Free energy diagrams of the ORR and OER on $\mathrm{CoN}_{4}$. spin-state transition in the case of $* \mathrm{O}_{2} \mathrm{H} / \mathrm{CoN}_{4}$ is essential for the exceptional ORR activity in acid. The ORR onset potential at $\mathrm{pH} 1$ would decrease to $0.54 \mathrm{~V} / \mathrm{RHE}$ if the $* \mathrm{O}_{2} \mathrm{H} / \mathrm{CoN}_{4}$ spin state remained in the LS state. For OER, $\mathrm{CoN}_{4}$ has an onset potential as high as $2.5 \mathrm{~V} / \mathrm{RHE}$ due to the weak adsorption of $* \mathrm{O}$ as a result of the rapid increase in the ${ }^{*} \mathrm{O}$ binding energy as a function of potential. On the other hand, $\mathrm{CoN}_{4}$ performs exceptionally in catalyzing the OER in alkaline media with an onset potential of $1.51 \mathrm{~V} / \mathrm{RHE}$. The weak adsorption of $* \mathrm{O}$ in acid is alleviated by operating at a much lower potential vs SHE due to the $\mathrm{pH}$ increase. The exceptional OER activity also benefits from the high capacitance of the $\mathrm{HS}$ state of $* \mathrm{OH} / \mathrm{CoN}_{4}$, which effectively stabilizes $* \mathrm{OH}$ adsorption at high potential. If $* \mathrm{OH} / \mathrm{CoN}_{4}$ was in the next favorable IS state, the onset potential would further increase to $1.7 \mathrm{~V} / \mathrm{RHE}$.

\section{CONCLUSION}

In summary, we show that the adsorption energies of reaction intermediates of the ORR and OER on the $\mathrm{CoN}_{4}$ center are strongly modulated by the applied potential. In addition, the spin crossover effect induced by the applied potential also plays an important role in determining the adsorption energies of reaction intermediates and thus the electrocatalytic activities of $\mathrm{CoN}_{4}{ }^{-} \mathrm{C}$ for the ORR and OER. We demonstrate that the spin crossover occurs because each spin state responds differently to the applied potential due to different capacitance and PZC.

\section{ASSOCIATED CONTENT}

\section{(s) Supporting Information}

The Supporting Information is available free of charge at https://pubs.acs.org/doi/10.1021/acscatal.0c02458.

Atomic models, potential-dependent adsorption energies of $* \mathrm{OH}$ and $* \mathrm{OOH}$ on $\mathrm{CoN}_{4}$, potential-dependent dorbital occupancies and structural evolution (PDF)

\section{AUTHOR INFORMATION}

\section{Corresponding Author}

Graeme Henkelman - Department of Chemistry and the Oden Institute for Computational Engineering and Sciences, The University of Texas at Austin, Austin, Texas 78712-0165, United States; $\odot$ orcid.org/0000-0002-0336-7153; Email: henkelman@utexas.edu

\section{Author}

Zhiyao Duan - Department of Chemistry and the Oden Institute for Computational Engineering and Sciences, The University of Texas at Austin, Austin, Texas 78712-0165, United States 
Complete contact information is available at:

https://pubs.acs.org/10.1021/acscatal.0c02458

\section{Notes}

The authors declare no competing financial interest.

\section{ACKNOWLEDGMENTS}

This work was supported by the Department of Energy under contract DE-SC0010576 and the Welch Foundation under grant F-1841. The calculations were done at the National Energy Research Scientific Computing Center and the Texas Advanced Computing Center, partially through the NSF XSEDE program.

\section{REFERENCES}

(1) Kraytsberg, A.; Ein-Eli, Y. Review of Advanced Materials for Proton Exchange Membrane Fuel Cells. Energy Fuels 2014, 28, 73037330.

(2) Tachibana, Y.; Vayssieres, L.; Durrant, J. R. Artificial photosynthesis for solar water-splitting. Nat. Photonics 2012, 6, 511-518.

(3) Huang, Z.-F.; Wang, J.; Peng, Y.; Jung, C.-Y.; Fisher, A.; Wang, $\mathrm{X}$. Design of Efficient Bifunctional Oxygen Reduction/Evolution Electrocatalyst: Recent Advances and Perspectives. Adv. Energy Mater. 2017, 7, 1700544.

(4) Chen, M.; He, Y.; Spendelow, J. S.; Wu, G. Atomically Dispersed Metal Catalysts for Oxygen Reduction. ACS Energy Lett. 2019, 4, 1619-1633.

(5) Liu, X.; Dai, L. Carbon-based metal-free catalysts. Nat. Rev. Mater. 2016, 1, 16064.

(6) Zhang, Q.; Guan, J. Mono-/Multinuclear Water Oxidation Catalysts. ChemSusChem 2019, 12, 3209-3235.

(7) Wu, G.; More, K. L.; Johnston, C. M.; Zelenay, P. HighPerformance Electrocatalysts for Oxygen Reduction Derived from Polyaniline, Iron, and Cobalt. Science 2011, 332, 443-447.

(8) Bai, L.; Duan, Z.; Wen, X.; Guan, J. Bifunctional atomic ironbased catalyst for oxygen electrode reactions. J. Catal. 2019, 378, 353-362.

(9) He, Y.; et al. Highly active atomically dispersed CoN4 fuel cell cathode catalysts derived from surfactant-assisted MOFs: carbon-shell confinement strategy. Energy Environ. Sci. 2019, 12, 250-260.

(10) Zhang, Q.; Duan, Z.; Li, M.; Guan, J. Atomic cobalt catalysts for the oxygen evolution reaction. Chem. Commun. 2020, 56, 794797.

(11) Guan, J.; Duan, Z.; Zhang, F.; Kelly, S.; Si, R.; Dupuis, M.; Huang, Q.; Chen, J.; Tang, C.; Li, C. Water oxidation on a mononuclear manganese heterogeneous catalyst. Nat. Catal. 2018, 1, 870-877.

(12) Bai, L.; Duan, Z.; Wen, X.; Si, R.; Guan, J. Atomically dispersed manganese-based catalysts for efficient catalysis of oxygen reduction reaction. Appl. Catal., B 2019, 257, 117930.

(13) Li, J.; et al. Atomically dispersed manganese catalysts for oxygen reduction in proton-exchange membrane fuel cells. Nat. Catal. 2018, $1,935-945$.

(14) Chung, H. T.; Cullen, D. A.; Higgins, D.; Sneed, B. T.; Holby, E. F.; More, K. L.; Zelenay, P. Direct atomic-level insight into the active sites of a high-performance PGM-free ORR catalyst. Science 2017, 357, 479-484.

(15) Zitolo, A.; Ranjbar-Sahraie, N.; Mineva, T.; Li, J.; Jia, Q.; Stamatin, S.; Harrington, G. F.; Lyth, S. M.; Krtil, P.; Mukerjee, S.; Fonda, E.; Jaouen, F. Identification of catalytic sites in cobaltnitrogen-carbon materials for the oxygen reduction reaction. Nat. Commun. 2017, 8, 957.

(16) Kattel, S.; Wang, G. A density functional theory study of oxygen reduction reaction on $\mathrm{Me}-\mathrm{N} 4(\mathrm{Me}=\mathrm{Fe}, \mathrm{Co}$, or $\mathrm{Ni})$ clusters between graphitic pores. J. Mater. Chem. A 2013, 1, 10790-10797.

(17) Kattel, S.; Wang, G. Reaction Pathway for Oxygen Reduction on FeN4 Embedded Graphene. J. Phys. Chem. Lett. 2014, 5, 452-456.
(18) Kattel, S.; Atanassov, P.; Kiefer, B. Catalytic activity of Co-Nx/ $\mathrm{C}$ electrocatalysts for oxygen reduction reaction: a density functional theory study. Phys. Chem. Chem. Phys. 2013, 15, 148-153.

(19) Nørskov, J. K.; Rossmeisl, J.; Logadottir, A.; Lindqvist, L.; Kitchin, J. R.; Bligaard, T.; Jonsson, H. Origin of the Overpotential for Oxygen Reduction at a Fuel-Cell Cathode. J. Phys. Chem. B 2004, 108, 17886-17892.

(20) Fu, C.; Liu, C.; Li, T.; Zhang, X.; Wang, F.; Yang, J.; Jiang, Y.; Cui, P.; Li, H. DFT calculations: A powerful tool for better understanding of electrocatalytic oxygen reduction reactions on Ptbased metallic catalysts. Comput. Mater. Sci. 2019, 170, 109202.

(21) Zhang, L.; Henkelman, G. Computational Design of AlloyCore@Shell Metal Nanoparticle Catalysts. ACS Catal. 2015, 5, 655660.

(22) Liu, K.; Qiao, Z.; Hwang, S.; Liu, Z.; Zhang, H.; Su, D.; Xu, H.; $\mathrm{Wu}, \mathrm{G}$.; Wang, G. Mn- and $\mathrm{N}$ - doped carbon as promising catalysts for oxygen reduction reaction: Theoretical prediction and experimental validation. Appl. Catal., B 2019, 243, 195-203.

(23) Kim, D.; Shi, J.; Liu, Y. Substantial Impact of Charge on Electrochemical Reactions of Two-Dimensional Materials. J. Am. Chem. Soc. 2018, 140, 9127-9131.

(24) Duan, Z.; Henkelman, G. Calculations of the pH-Dependent Onset Potential for $\mathrm{CO}$ Electrooxidation on $\mathrm{Au}(111)$. Langmuir 2018, 34, 15268-15275.

(25) Duan, Z.; Henkelman, G. Theoretical Resolution of the Exceptional Oxygen Reduction Activity of $\mathrm{Au}(100)$ in Alkaline Media. ACS Catal. 2019, 9, 5567-5573.

(26) Gütlich, P.; Goodwin, H.; Garcia, Y.Spin Crossover in Transition Metal Compounds I; Springer: 2004; Spin Crossover in Transition Metal Compounds, pp 1-47.

(27) Diefenbach, M.; Kim, K. Towards Molecular Magnetic Switching with an Electric Bias. Angew. Chem., Int. Ed. 2007, 46, 7640-7643

(28) Baadji, N.; Piacenza, M.; Tugsuz, T.; Sala, F.; Maruccio, G.; Sanvito, S. Electrostatic spin crossover effect in polar magnetic molecules. Nat. Mater. 2009, 8, 813-817.

(29) Kresse, G.; Hafner, J. Ab Initio Molecular Dynamics for Liquid Metals. Phys. Rev. B: Condens. Matter Mater. Phys. 1993, 47, 558.

(30) Kresse, G.; Furthmüller, J. Efficiency of Ab-Initio Total Energy Calculations for Metals and Semiconductors Using a Plane-Wave Basis Set. Comput. Mater. Sci. 1996, 6, 15-50.

(31) Kresse, G.; Furthmüller, J. Efficient Iterative Schemes for $\mathrm{Ab}$ Initio Total-Energy Calculations Using a Plane-Wave Basis Set. Phys. Rev. B: Condens. Matter Mater. Phys. 1996, 54, 11169.

(32) Perdew, J. P.; Burke, K.; Ernzerhof, M. Generalized Gradient Approximation Made Simple. Phys. Rev. Lett. 1996, 77, 3865-3868.

(33) Blöchl, P. E. Projector augmented-wave method. Phys. Rev. B: Condens. Matter Mater. Phys. 1994, 50, 17953-17979.

(34) Dudarev, S. L.; Botton, G. A.; Savrasov, S. Y.; Humphreys, C. J.; Sutton, A. P. Electron-energy-loss spectra and the structural stability of nickel oxide: An LSDA+U study. Phys. Rev. B: Condens. Matter Mater. Phys. 1998, 57, 1505-1509.

(35) Wang, L.; Maxisch, T.; Ceder, G. Oxidation energies of transition metal oxides within the GGA + U framework. Phys. Rev. B: Condens. Matter Mater. Phys. 2006, 73, 195107.

(36) Monkhorst, H. J.; Pack, J. D. Special Points for Brillouin-Zone Integrations. Phys. Rev. B 1976, 13, 5188-5192.

(37) Allen, J. P.; Watson, G. W. Occupation matrix control of d- and f-electron localisations using DFT + U. Phys. Chem. Chem. Phys. 2014, 16, 21016-21031.

(38) Mathew, K.; Sundararaman, R.; Letchworth-Weaver, K.; Arias, T. A.; Hennig, R. G. Implicit Solvation Model for Density-Functional Study of Nanocrystal Surfaces and Reaction Pathways. J. Chem. Phys. 2014, 140, No. 084106.

(39) Fishman, M.; Zhuang, H. L.; Mathew, K.; Dirschka, W.; Hennig, R. G. Accuracy of Exchange-Correlation Functionals and Effect of Solvation on the Surface Energy of Copper. Phys. Rev. B: Condens. Matter Mater. Phys. 2013, 87, 245402. 
(40) Taylor, C. D.; Wasileski, S. A.; Filhol, J.-S.; Neurock, M. First Principles Reaction Modeling of the Electrochemical Interface: Consideration and Calculation of a Tunable Surface Potential from Atomic and Electronic Structure. Phys. Rev. B: Condens. Matter Mater. Phys. 2006, 73, 165402.

(41) Filhol, J.-S.; Neurock, M. Elucidation of the Electrochemical Activation of Water over Pd by First Principles. Angew. Chem., Int. Ed. 2006, 45, 402-406.

(42) Hansen, H. A.; Rossmeisl, J.; Nørskov, J. K. Surface Pourbaix Diagrams and Oxygen Reduction Activity of $\mathrm{Pt}, \mathrm{Ag}$ and $\mathrm{Ni}(111)$ Surfaces Studied by DFT. Phys. Chem. Chem. Phys. 2008, 10, 3722.

(43) Luryi, S. Quantum capacitance devices. Appl. Phys. Lett. 1988, 52, 501-503.

(44) Ochoa-Calle, A.; Guevara-García, A.; Vazquez-Arenas, J.; González, I.; Galván, M. Establishing the Relationship between Quantum Capacitance and Softness of N-Doped Graphene/Electrolyte Interfaces within the Density Functional Theory Grand Canonical KohnSham Formalism. J. Phys. Chem. A 2020, 124, 573-581. 